\title{
Optimized operation of dielectric laser accelerators: Single bunch
}

\author{
Adi Hanuka* and Levi Schächter \\ Department of Electrical, Engineering Technion-Israel Institute of Technology, Haifa 32000, Israel
}

(Received 11 September 2017; published 9 May 2018)

\begin{abstract}
We introduce a general approach to determine the optimal charge, efficiency and gradient for laser driven accelerators in a self-consistent way. We propose a way to enhance the operational gradient of dielectric laser accelerators by leverage of beam-loading effect. While the latter may be detrimental from the perspective of the effective gradient experienced by the particles, it can be beneficial as the effective field experienced by the accelerating structure, is weaker. As a result, the constraint imposed by the damage threshold fluence is accordingly weakened and our self-consistent approach predicts permissible gradients of $\sim 10 \mathrm{GV} / \mathrm{m}$, one order of magnitude higher than previously reported experimental results-with unbunched pulse of electrons. Our approach leads to maximum efficiency to occur for higher gradients as compared with a scenario in which the beam-loading effect on the material is ignored. In any case, maximum gradient does not occur for the same conditions that maximum efficiency does-a trade-off set of parameters is suggested.
\end{abstract}

DOI: 10.1103/PhysRevAccelBeams.21.054001

\section{INTRODUCTION}

Today's electron accelerators are predominantly driven by rf sources. Being structure-based systems, operating with the lowest electromagnetic mode, the accelerating gradient is limited by the breakdown in the metal's surface [1]. However, in the optical regime it has been experimentally shown [2,3] that dielectric materials held higher fields before breakdown. Therefore, it indicates what should be the general trend, namely operating at sub-mm or optical wavelengths, as this allows higher accelerating gradients. For example, the Stanford Linear Collider's gradient is of the order of $20 \mathrm{MeV} / \mathrm{m}$ [4], while in an optical accelerator 50 times this value is anticipated [5].

Another profound difference between a laser driven accelerator as compared with its microwave counterpart is their material. At optical frequencies Ohm loss makes metals prohibitively lossy. Thus, low loss dielectric materials are virtually the only alternative for an accelerating structure, regardless of whether the latter is used as an optical electron collider [6], a possible light source [7], or as a module for medical devices [8]. Throughout the years several dielectric structures have been proposed [9-12] and more recently experimental results were reported [13-15]. In all these configurations fluence damage [16] is a limiting

\footnotetext{
*Adiha@tx.technion.ac.il
}

Published by the American Physical Society under the terms of the Creative Commons Attribution 4.0 International license. Further distribution of this work must maintain attribution to the author(s) and the published article's title, journal citation, and DOI. factor, whereas in rf machines, breakdown at the metalvacuum interface is a critical impediment [17].

Both rf and laser accelerators have an important phenomenon in common, this is the beam loading. It results from the wakefield generated by each particle [18], thus reducing the effective gradient experienced by the same or trailing particles. As shown subsequently, the field reduction may be beneficial, since the structure is exposed to a weaker electromagnetic field.

In the framework of this paper we present a general approach relevant to any guided-mode or resonant Floquet harmonic in a dielectric laser-driven acceleration structure, which aims to achieve a self-consistent analysis of the optimal charge, gradient, and efficiency. Contrary to the approach in Ref. [19], we take into account the short range wakefield only once. But in addition, we account for its effect on the dielectric material too. Proper design of the operation parameters, indicates that beam-loading although reduces the effective gradient experienced by the particles, it also enlarges the gap between the maximum field experienced by the material and the damage threshold fluence. As a result, the laser power may be increased and so is the amount of accelerated charge. The optimization process developed predicts an unloaded gradient of $\sim 10 \mathrm{GV} / \mathrm{m}$. This is one order of magnitude higher than previous experiments demonstrated $[14,15]$.

\section{SYSTEM DESCRIPTION}

Let us now introduce the general approach. Common to all various structures is the vacuum channel, where the electrons propagate, and the single $\mathrm{TM}_{01}$ mode that accelerates them. Although preliminary results were 


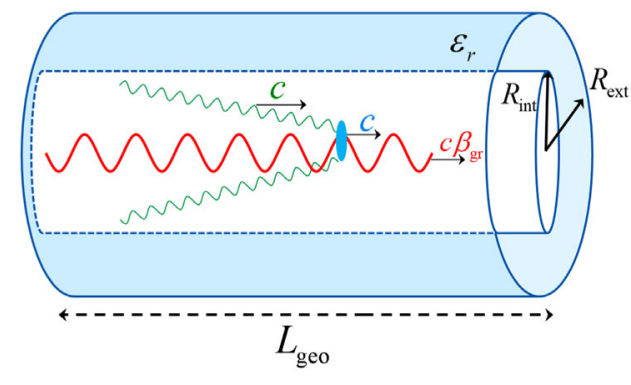

FIG. 1. Schematics of the basic principle and envisaged geometry. Single relativistic microbunches (blue) is accelerated by $\mathrm{TM}_{01}$ laser mode (red) which propagates at $c \beta_{\mathrm{gr}}$ group velocity, in a vacuum channel of a dielectric $\left(\varepsilon_{\mathrm{r}}\right)$ loaded cylinder. The wake (green), generated by the bunch, propagates at its velocity [22] along the interaction region $L_{\text {geo }}$.

previously presented for planar Bragg structures [20], for the numerical examples presented subsequently in this study, we adopt an azimuthally symmetric dielectric-loaded waveguide [21], as shown in Fig. 1, since it is possible to evaluate all the quantities of interest analytically. However, while the group velocity, interaction impedance, and wake parameters may vary from one configuration to another, the general trend is expected to be the same. We anticipate the same quantities to control the interaction whether it is a guided mode or synchronous harmonic (open structure).

Specifically, the fields components of a $\mathrm{TM}_{01}$ mode which propagates at a phase velocity equals to the speed of light in a cylindrical vacuum channel are

$$
\begin{aligned}
E_{z} & =G_{0} \exp \left(-j \frac{\omega_{\mathrm{L}}}{c} z\right) \\
E_{r} & =\frac{j}{2} \frac{\omega_{\mathrm{L}}}{c} r G_{0} \exp \left(-j \frac{\omega_{\mathrm{L}}}{c} z\right) \\
H_{\phi} & =\frac{j}{2 \eta_{0}} \frac{\omega_{\mathrm{L}}}{c} r G_{0} \exp \left(-j \frac{\omega_{\mathrm{L}}}{c} z\right)
\end{aligned}
$$

where $G_{0}$ is the accelerating unloaded gradient, $\omega_{\mathrm{L}}$ is laser's angular frequency, and $\eta_{0}$ is the wave impedance in vacuum. The idealized acceleration structure consists of a dielectric $\left(\varepsilon_{r}\right)$ loaded waveguide, whereby for a given dielectric (fused Silica) and vacuum channel radius $\left(R_{\text {int }}\right)$, the external radius $\left(R_{\text {ext }}\right)$ is set by imposing single mode $\left(\mathrm{TM}_{01}\right)$ operation and phase velocity equal to the speed of light in vacuum [see Eq. (B3)]. Moreover, note that for a given dielectric material $\varepsilon_{\mathrm{r}}$, imposing the group velocity sets $R_{\text {int }}$ and vice versa.

Additionally, more assumptions are at the foundation of our model: (i) the microbunch is a point-charge, and remains so all along the interaction region. (ii) The space-charge effect inside each microbunch is ignored. (iii) The laser pulse has a sharp rise/fall time relative to one laser period, and (iv) the conversion from a propagating laser mode to an accelerating $\mathrm{TM}_{01}$ mode (coupling process) is considered to be ideal. Each one of these idealizations may reduce the optimal charge, the gradient experienced by the electrons and of course the overall efficiency of the system, however these are beyond the scope of this study. (v) Fluence damage threshold is the only concern here and we ignore nonlinear optical effects (Kerr [23], Brillouin, Stokes, etc.). (vi) Since the focus of this study is the behavior of the field near the vacuum-dielectric interface, we ignore the fluence damage threshold of the confining metal. We wish to reiterate that the metallic boundary is not intrinsic to the operation, but rather allows us to readily determine the interaction impedance, wakefield and group velocity, for the sake of showcasing an analytical example for our general formulation. (vii) In case of single bunch operation, both the laser pulse and the wake leave the accelerating module before the next laser pulse fills in.

Previous studies $[6,19,20]$ formulated the efficiency of accelerating single bunch $\left(\eta_{1}\right)$ with a charge $q$ in terms of three quantities: (i) the accelerating unloaded gradient generated by the laser $\left(E_{\mathrm{acc}}=G_{0}\right)$; (ii) the wake coefficient $(\kappa)$ which by virtue of linearity of Maxwell's equations relates the decelerating electric field that acts on the bunch $\left(E_{\text {wake }}=\kappa q\right)$, and (iii) $\kappa_{1}$ is the projection of $\kappa$ on the fundamental (accelerating) mode. In these studies it was tacitly assumed that the maximum gradient applicable is limited by the damage threshold fluence-ignoring beamreduction of the field experienced by the dielectric. In the next paragraph we explain how we suggest to consider this effect, which would be referred to as the reduced field.

Virtually in all relevant acceleration structures the energy flux $\left(S_{z}=E_{r} H_{\phi}^{*} / 2\right)$ reaches its maximum at the vacuumdielectric interface

$$
S_{\mathrm{z}, \max }\left(r=R_{\mathrm{int}}+0\right)=\frac{1}{2 \eta_{0} \varepsilon_{r}}\left[\frac{1}{2} \frac{\omega_{\mathrm{L}}}{c} R_{\mathrm{int}} G_{0}\right]^{2}
$$

where $\varepsilon_{r}$ is the dielectric coefficient adjacent to the vacuum channel, and $R_{\text {int }}$ is the radius of the latter. We wish to emphasize that this is the flux of the $\mathrm{TM}_{01}$ mode in the dielectric in terms of the accelerating gradient $\left(G_{0}\right)$, in the absence of the electron beam. However, since we examine the effect of the electrons on the fundamental mode, we shall consider an effective electric field $\left(E_{\text {eff }}\right)$. Note that the effective electric field in the material near the dielectricvacuum interface is $E_{\text {eff }}=\sqrt{E_{\mathrm{r}}^{2}+E_{z}^{2}}$. Given a single mode operation $\omega_{\mathrm{L}} R_{\text {int }} /\left(2 \varepsilon_{\mathrm{r}} c\right)<1$, the maximum effective field in the material is approximately $E_{\text {eff }} \simeq E_{z}$.

Since the phase velocity is $c$, the latter is $G_{0}$ reduced by the projection $\left(\kappa_{1}\right)$ of the wakefield solely on the accelerating mode, ignoring the contribution of all the other modes. Consequently, the maximum energy flux in the dielectric is assumed to be given by

$$
S_{\mathrm{z}, \max }\left(r=R_{\mathrm{int}}+0\right)=\frac{1}{2 \eta_{0} \varepsilon_{r}}\left[\frac{1}{2} \frac{\omega_{\mathrm{L}}}{c} R_{\mathrm{int}} E_{\mathrm{eff}}\right]^{2}
$$

or explicitly, the effective field in the reduced case (superscript R) is $E_{\text {eff }}^{(\mathrm{R})} \equiv G_{0}-\kappa_{1} q$. Note that the latter quantity is defined in the dielectric. Evidently, if the reduction $\kappa_{1} q$ is 
ignored, namely $E_{\text {eff }}^{(\mathrm{UR})} \equiv G_{0}$, in what follows we refer to this incidence as the unreduced case (superscript UR).

It is important to emphasize that the projection of the wake on the fundamental mode is proportional to the value $2 h(\tau)$ whereby $h(\tau)$ is the Heaviside step function. In this study we consider a conservative scenario whereby the typical value corresponds to the front of the wake rather than the main trailing wake. If we consider the latter, the reduction leads to a better optimization but we prefer the conservative route rather than the best case scenario.

Given the flattop laser pulse duration $\tau_{\mathrm{p}}$, the maximum Poynting vector and the fluence are related $S_{\max }\left(R_{\text {int }}+0\right)=$ $F\left(\tau_{\mathrm{p}}\right) / \tau_{\mathrm{p}}$. Therefore substituting the latter to Eq. (3), the effective field experienced by the dielectric is

$$
E_{\text {eff }}=\frac{\sqrt{2 \eta_{0} \varepsilon_{r}}}{\frac{1}{2} \frac{\omega_{\mathrm{L}}}{c} R_{\mathrm{int}}} \sqrt{\frac{F\left(\tau_{\mathrm{p}}\right)}{\tau_{\mathrm{p}}}} .
$$

Furthermore, we assume that the fluence dependence on the pulse duration is known and determined by $F\left(\tau_{\mathrm{p}}\right)$; explicitly, in the examples presented in this study, we adopt the expression in Ref. [24] which is a parametrization of the measured data presented in Ref. [16]

$$
F\left[\frac{J}{\mathrm{~cm}^{2}}\right]= \begin{cases}1.44 \tau_{\mathrm{p}}^{1 / 2} & \tau_{\mathrm{p}}[p \mathrm{sec}]>10 \\ 2.51 \tau_{\mathrm{p}}^{1 / 4} & 0.4<\tau_{\mathrm{p}}[p \mathrm{sec}]<10 . \\ 2 & \tau_{\mathrm{p}}[p \mathrm{sec}]<0.4\end{cases}
$$

Note that although this fluence formula is based on experimental results of laser wavelength $\lambda_{\mathrm{L}}=1 \mu \mathrm{m}$, we adopt it also for $\lambda_{\mathrm{L}}=2 \mu \mathrm{m}$, due to lack of experimental data with the latter. Next, we introduce one more condition: full overlap between the laser pulse that propagates at $c \beta_{\mathrm{gr}}$ and a relativistic single microbunch along the interaction length, $L_{\mathrm{geo}}$. This condition is satisfied by setting the pulse duration, which is equal to the delay time $\tau_{\mathrm{D}}[6]$, to be

$$
\tau_{\mathrm{p}}=\tau_{\mathrm{D}} \equiv \frac{L_{\mathrm{geo}}}{c}\left(\beta_{\mathrm{gr}}^{-1}-1\right)
$$

While the group velocity is an electromagnetic property of the structure, the interaction length is determined by two parameters: the energy gain required $\Delta \gamma_{\text {acc }}$ and the effective loaded gradient that acts on the charge $G_{\text {Loaded }}=G_{0}-\kappa q$. Therefore, the geometric length is given by

$$
L_{\mathrm{geo}}=\frac{\Delta \gamma_{\mathrm{acc}} m_{\mathrm{e}} c^{2}}{e G_{\text {Loaded }}}=\frac{\Delta \gamma_{\mathrm{acc}} m_{\mathrm{e}} c^{2}}{e\left(G_{0}-\kappa q\right)}
$$

where $m_{\mathrm{e}}$ and $e$ are the electron's mass and charge respectively. It is important to emphasize the difference between $G_{\text {loaded }}$ and $E_{\text {eff }}$, the former is the electric field acting on the particle whereas the latter is the electric field that the structure is exposed to at the vacuum-dielectric interface.

At this stage, considering Eqs. (4)-(7), the unloaded gradient $G_{0}$ and charge $q$ are yet unknown. However, the inter-dependence between the parameters in Eqs. (4)-(7) results in a transcendental equation for $G_{0}$ and $q$. Given the five free parameters: normalized energy gain $\left(\Delta \gamma_{\text {acc }}\right)$, the vacuum channel's radius $\left(R_{\text {int }}\right)$, the group velocity $\left(\beta_{\mathrm{gr}}\right)$, the laser wavelength $\left(\lambda_{\mathrm{L}}\right)$, and the geometrical length of the structure $\left(L_{\text {geo }}\right)$, we can determine the gradient given the charge or vice versa, we can calculate the charge given the gradient. In either one of the options the solution is selfconsistent. Given the solution for the gradient and charge, the efficiency $\left(\eta_{1}=4 \kappa_{1} q G_{0}^{-1}\left(1-\kappa q G_{0}^{-1}\right)\right)$ can be evaluated. Moreover, we show in what follows that optimal values exist for the efficiency as well gradient.

\section{SELF-CONSISTENT ANALYSIS}

A flavor of the general trend of such a self-consistent solution, for a single bunch in the reduced case, is given in Fig. 2 for the parameters in Table I and a range of interaction length values $\left(L_{\text {geo }}\right)$. While a detailed comparison between the unreduced and reduced cases is given in Sec. IV, for the sake of simplicity, in what follows we focus on the properties of the latter.

Figure 2 reveals a monotonic variation of the charge (dashed blue), whereas both the efficiency (red) and unloaded gradient (dotted green) reach maximum. However, the conditions for maximum efficiency and maximum unloaded gradient cannot be satisfied simultaneously. Moreover, as shown in Fig. 2, it is advantageous to operate in short structures, where higher gradients are available. For example, an unloaded gradient of $G_{0} \simeq$ $9 \mathrm{GV} / \mathrm{m}$ and loaded gradient of $G_{\text {Loaded }} \simeq 3 \mathrm{GV} / \mathrm{m}$ are

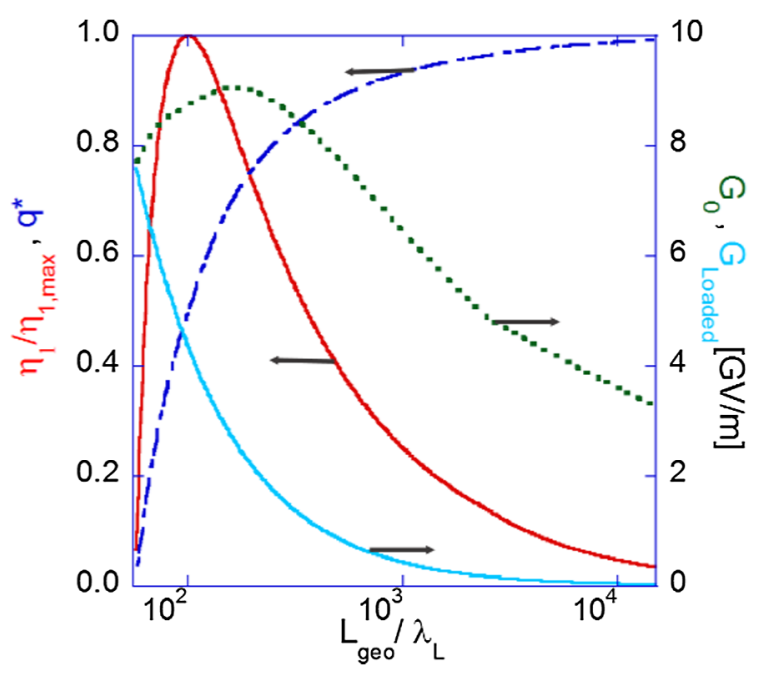

FIG. 2. Reduced case quantities for single bunch as a function of geometrical length. Left vertical axis: normalized charge $q^{*}=$ $\kappa q / G_{0}$ (dashed blue), efficiency normalized to its maximum $\eta_{1 \text {, max }}$ (red) — see derivation in Appendix B. Right vertical axis: unloaded gradient (turquoise) and loaded gradient (dotted green). While the charge varies monotonically, both the efficiency and unloaded gradient reach maximum. Notably, maximum efficiency and maximum gradient $\left(G_{0}\right)$ occur for different geometrical lengths, and therefore cannot be satisfied together. 
TABLE I. Parameters of the laser and the envisaged structure shown in Fig. 1. (*) Average laser power formula is given by $P_{\mathrm{L}}=G_{0}^{2} \lambda_{\mathrm{L}}^{2} / Z_{\text {int }}$.

\begin{tabular}{lcc}
\hline \hline Parameter & Symbol & Value \\
\hline Laser & & \\
Laser wavelength $[\mu \mathrm{m}]$ & $\lambda_{\mathrm{L}}$ & 2 \\
Group velocity & $\beta_{\mathrm{gr}}$ & 0.63 \\
Phase velocity & $\beta_{\mathrm{ph}}$ & 1.0 \\
Interaction impedance $[\Omega]$ & $Z_{\mathrm{int}}$ & 370 \\
Laser power $[\mathrm{kW}]$ & $P_{\mathrm{L}}^{*}$ & $10.8 \cdot\left\{G_{0}\left[\frac{\mathrm{GV}}{\mathrm{m}}\right]\right\}^{2}$ \\
Structure & & 0.5 \\
Internal radius $\left[\lambda_{\mathrm{L}}\right]$ & $R_{\mathrm{int}}$ & 0.65 \\
External radius $\left[\lambda_{\mathrm{L}}\right]$ & $R_{\mathrm{ext}}$ & 2.1 \\
Dielectric constant & $\varepsilon_{\mathrm{r}}$ & 18 \\
Wake coefficient $[\mathrm{GV}]$ & $\kappa$ & 1.7 \\
Energy gain required & $\Delta \gamma_{\mathrm{acc}}$ & 67 \\
Maximum efficiency $[\%]$ & $\eta_{1, \max }$ & \\
\hline \hline
\end{tabular}

feasible in a $0.3 \mathrm{~mm}$ long structure with an efficiency of $0.58 \eta_{1, \max }$. However, for a relatively long interaction length $\left(L_{\text {geo }}=6 \mathrm{~mm}\right)$, the maximum unloaded gradient and charge are $G_{0}=4.7 \mathrm{GV} / \mathrm{m}, q=0.25 \mathrm{pC}$ respectively, therefore the loaded gradient is $G_{\text {Loaded }}=0.145 \mathrm{GV} / \mathrm{m}$ and the efficiency is $0.08 \eta_{1, \max }$.

As could be inferred from Eq. (6), when the bunch enters the structure the laser pulse might be half a way in the structure, while no wake has been generated in this region to reduce the exposure of the dielectric to the intense optical field. During this time period $\left(\tau_{\mathrm{D}}\right)$, the field at the dielectric interface may be higher than the damage threshold fluence (DTF). Therefore, the latter would set an upper limit on the unloaded gradient values $\left(G_{0}\right)$. Figure 3 reveals the DTF gradient $\left(\mathrm{G}_{\mathrm{DTF}}\right)$ dependence on the delay time $\left(\tau_{\mathrm{D}}\right)$, and the

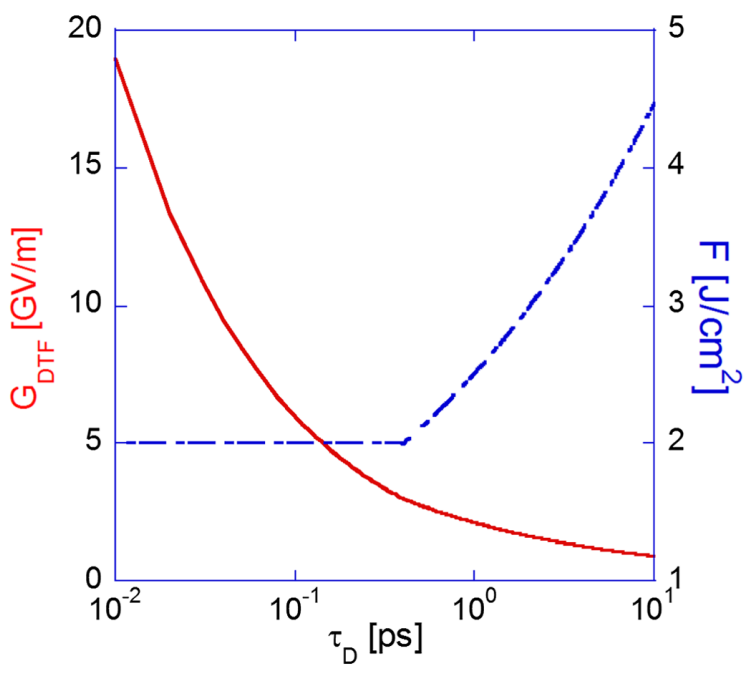

FIG. 3. Damage threshold fluence gradient (red) and the corresponding fluence values (dashed blue) in Eq. (5) as a function of delay time. Since $\mathrm{G}_{\mathrm{DTF}}$ set an upper limit on the unloaded gradient values, it is advantageous to reduce the delay time. corresponding fluence values $(F)$ presented in Eq. (5). Therefore, the fact that the delay time might be of the order of picoseconds, would set an upper limit on the unloaded gradient values. Consequently, it is advantageous to reduce the delay time by either shortening the structure or operating with a higher group velocity.

Before we proceed, we would like to note that since dielectric breakdown is nonrecoverable, a failure at the injector may cause the accelerator structure to be destroyed by high-intensity laser fields. Therefore, it might be critical to build a control system that would block the laser field in case the electron bunch does not arrive at the structure, due to some upstream fault, similar to control systems of electron damping rings.

\section{DISCUSSION}

At this stage, we may compare between the reduced case, in which the beam loading effect on the dielectric is considered, to the unreduced case, in which the effect is ignored. The four constraints formulated in Eqs. (4)-(7) reduce the number of free parameters to five: the radius of the vacuum channel $R_{\text {int }}$, the structure's geometric length $L_{\text {geo }}$, the group velocity $\beta_{\text {gr }}$, the required energy gain $\Delta \gamma_{\text {acc }}$, and the driving laser's frequency $\omega_{\mathrm{L}}$. The solution, expressed in terms of the charge in a microbunch for the unreduced case, is given by

$$
q^{(\mathrm{UR})}=\frac{1}{\kappa}\left[\sqrt{\frac{2 \eta_{0} \varepsilon_{r} F\left(\tau_{\mathrm{p}}\right)}{\left(\frac{1}{2} \frac{\omega_{\mathrm{L}}}{c} R_{\mathrm{int}}\right)^{2} \tau_{\mathrm{p}}}}-\frac{\Delta \gamma_{\mathrm{acc}} m_{\mathrm{e}} c^{2}}{e L_{\mathrm{geo}}}\right]
$$

where the pulse duration is $\tau_{\mathrm{p}}=L_{\mathrm{geo}}\left(\beta_{\mathrm{gr}}^{-1}-1\right) / c$, and $F\left(\tau_{\mathrm{p}}\right)$ is the fluence. Both the wake coefficient $\kappa$, and its

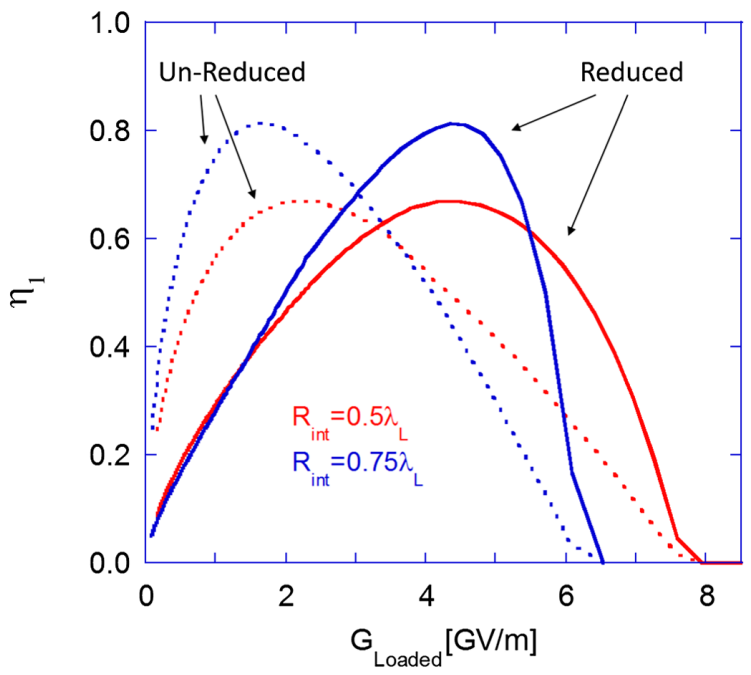

FIG. 4. Single bunch efficiency vs loaded gradient for two different radii in each case: reduced (solid curves) and unreduced (dashed curves). Although maximum efficiency is the same in both cases, it occurs for higher loaded gradients in the reduced case. Also, wider vacuum channel presents higher efficiency. 
TABLE II. Comparison between reduced (R) and un-reduced (UR) cases for the parameters in Table I and three scenarios.

\begin{tabular}{|c|c|c|c|c|c|c|c|c|}
\hline \multirow[b]{2}{*}{ Parameter } & \multirow[b]{2}{*}{ Symbol } & \multirow[b]{2}{*}{ Units } & \multicolumn{2}{|c|}{ I } & \multicolumn{2}{|c|}{ II } & \multicolumn{2}{|c|}{ III } \\
\hline & & & (R) & (UR) & $(\mathrm{R})$ & (UR) & $(\mathrm{R})$ & (UR) \\
\hline Structure length & $L_{\mathrm{geo}}$ & $\mathrm{mm}$ & \multicolumn{2}{|c|}{0.12} & \multicolumn{2}{|c|}{0.2} & \multicolumn{2}{|c|}{5.2} \\
\hline Pulse duration & $\tau_{\mathrm{p}}$ & ps & \multicolumn{2}{|c|}{0.23} & \multicolumn{2}{|c|}{0.38} & \multicolumn{2}{|c|}{10} \\
\hline Microbunch charge & $q$ & $\mathrm{fC}$ & 37 & 12 & 240 & 80 & 257 & 85 \\
\hline Efficiency & $\eta_{1}$ & $\eta_{1, \max }$ & 0.3 & 0.1 & 1.0 & 0.75 & 0.14 & 0.36 \\
\hline Unloaded gradient & $G_{0}$ & $\mathrm{GV} / \mathrm{m}$ & 7.9 & 7.45 & 8.6 & 5.7 & 4.7 & 1.7 \\
\hline Loaded gradient & $G_{\text {Loaded }}$ & $\mathrm{GV} / \mathrm{m}$ & \multicolumn{2}{|c|}{7.3} & \multicolumn{2}{|c|}{4.3} & \multicolumn{2}{|c|}{0.17} \\
\hline
\end{tabular}

first mode's weight $W_{1} \equiv \kappa_{1} / \kappa$ depend on the vacuum channel radius and the group velocity $\beta_{\mathrm{gr}}$.

According to the set of equations for the reduced case, we can show that the charge in a microbunch is

$$
q^{(\mathrm{R})}=\frac{1}{1-W_{1}} q^{(\mathrm{UR})} .
$$

Next, given the charge, the gradient $G_{0}$ is calculated based on Eq. (4) or Eq. (7). Therefore, as shown in Appendix C, in both cases (un-reduced and reduced) the loaded gradient is the same (e.g. $\left.G_{\text {Loaded }}^{(\mathrm{UR})}=G_{\text {Loaded }}^{(\mathrm{R})}\right)$, although occurring for different geometric length. Figure 4 shows the efficiency as a function of the loaded gradient for two vacuum channel radii $-0.75 \lambda_{\mathrm{L}}$ (blue) and $0.5 \lambda_{\mathrm{L}}$ (red), and two cases for each radii - reduced (solid curves) and unreduced (dotted curves). First, it is evident that higher maximum efficiency $(81 \%$ vs $67 \%)$ occurs for wider vacuum channel $\left(0.75 \lambda_{\mathrm{L}}\right.$ vs $\left.0.5 \lambda_{\mathrm{L}}\right)$. Second, maximum efficiency occurs for higher loaded gradient in the reduced case as compared with the unreduced case. For example, for $R_{\text {int }}=0.5 \lambda_{\mathrm{L}}, 67 \%$ maximum efficiency occurs for loaded gradient of $5 \mathrm{GV} / \mathrm{m}$ in the reduced case and only $2 \mathrm{GV} / \mathrm{m}$ in the un-reduced case.

In what follows we show that it is advantageous to operate with short (sub mm) structures since the loaded gradient is anticipated to be higher. Table II presents three scenarios (corresponding to different structure lengths), each of which regards both the reduced and unreduced cases. Explicitly, for short structures (scenarios I and II wherein $L_{\text {geo }} \leq 0.2 \mathrm{~mm}$ ), the loaded gradient is one order of magnitude higher than the long structure (scenario III wherein $L_{\text {geo }}=5.2 \mathrm{~mm}$ ). Also, for long structures a variety of other problematic effects arise such as laser defocusing.

Although the loaded gradient is the same for both the reduced and unreduced cases, the former is preferable in terms of microbunch charge and efficiency. The microbunch charge is nearly three times higher in the reduced case for all three scenarios, implying that the unloaded gradient is higher as well. For instance, in a $0.2 \mathrm{~mm}$ long structure, $q=240 \mathrm{fC}$ and $G_{0}=8.6 \mathrm{GV} / \mathrm{m}$ in the reduced case as compared with $q=80 \mathrm{fC}$ and $G_{0}=5.7 \mathrm{GV} / \mathrm{m}$ in the un-reduced case. Moreover, for the sub $\mathrm{mm}$ structures, the reduced case efficiency is higher than the unreduced case. For example, in a $0.2 \mathrm{~mm}$ long structure, the reduce case efficiency is $25 \%$ higher than the unreduced case. Such high efficiency leads to high charge, a total of $1.5 \times$ $10^{6}$ electrons in a bunch. In order to employ the single bunch theory developed in the current paper, in Ref. [25] we present three different regimes of operation for high energy physics applications: maximum efficiency, maximum charge, and maximum loaded gradient. We demonstrated the tradeoffs between the regimes, that result in loaded gradients of 1 to $6[\mathrm{GV} / \mathrm{m}]$, efficiencies of $20 \%$ to $80 \%$, and electrons flux of $10^{14}$ [el/sec], without significant concerns regarding damage threshold fluence.

\section{CONCLUSION}

In conclusion, we solved a self-consistent set of nonlinear constraints, taking into account the beam loading reduction on the material at the dielectric-vacuum interface. This case, which was referred to as the reduced case, presents unloaded gradient of $\sim 10 \mathrm{GV} / \mathrm{m}$. While the loaded gradient is anticipated to be the same in both cases (reduced and un-reduced), the maximum efficiency (and higher amount of charge) occur for a higher loaded gradient in the reduced case $(5 \mathrm{GV} / \mathrm{m})$ as compared with the unreduced case $(2 \mathrm{GV} / \mathrm{m})$.

Furthermore, we showed that it is advantageous to operate in sub millimeter structures, where higher gradients are available and are facilitated by shorter laser pulse and consequently by higher damage threshold fluence gradient $\left(G_{\mathrm{DTF}}\right)$.

In addition, maximum gradient does not occur for the same conditions that maximum efficiency occurs. However, the latter, together with the laser field, determine the optimal charge to be accelerated, a total of $\sim 10^{6}$ electrons in a bunch.

\section{ACKNOWLEDGMENTS}

This study was supported by Israel Science Foundation and Rothschild Caesarea Foundation.

\section{APPENDIX A: ENERGY GAIN DEPENDENCE ON THE GROUP VELOCITY}

In this Appendix we investigate the energy gain dependence on the group velocity, fluence, and structure's length 
TABLE III. Typical values of energy gain and loaded gradient for three cases, each case corresponds to a different regime of the fluence.

\begin{tabular}{lcccc}
\hline \hline Parameter & Symbol & A & B & C \\
\hline Structure length $[\mathrm{mm}]$ & $L_{\text {geo }}$ & 0.1 & 1 & 10 \\
Fluence $\left[\mathrm{J} / \mathrm{cm}^{2}\right]$ & $F$ & 2 & 3 & 6.3 \\
Energy gain required & $\Delta \gamma_{\text {acc }}$ & 1.6 & 6.6 & 32 \\
Loaded gradient $\left[\frac{\mathrm{GV}}{\mathrm{m}}\right]$ & $G_{\text {Loaded }}$ & 8.2 & 3.4 & 1.6 \\
\hline \hline
\end{tabular}

for the reduced case. Let us assume that the wake's first mode is dominant $\left(\kappa \simeq \kappa_{1}\right)$, and the pulse duration is $\tau_{\mathrm{p}}=\tau_{\mathrm{D}}$. The latter condition occurs for either a single bunch or for a short train of microbunches duration as compared with the delay time $\left(\tau_{\mathrm{B}} \ll \tau_{\mathrm{D}}\right)$. Therefore the energy gain required is

$$
\Delta \gamma_{\mathrm{acc}} \simeq \frac{\sqrt{2 \eta_{0} \varepsilon_{\mathrm{r}} e^{2} c\left(\beta_{\mathrm{gr}}^{-1}-1\right)^{-1}}}{\frac{1}{2} \frac{\omega_{\mathrm{L}}}{c} R_{\mathrm{int}} m_{\mathrm{e}} c^{2}} \sqrt{F\left(\tau_{\mathrm{D}}\right) L_{\mathrm{geo}}} .
$$

By substituting Eq. (5) and the parameters in Table I, we get

$$
\Delta \gamma_{\mathrm{acc}}= \begin{cases}5.73 L_{\mathrm{geo}}^{3 / 4} & L_{\mathrm{geo}}[\mathrm{mm}]>3 \\ 6.57 L_{\mathrm{geo}}^{5 / 8} & 0.12<L_{\mathrm{geo}}[\mathrm{mm}]<3 ; \\ 5.05 L_{\mathrm{geo}}^{1 / 2} & L_{\mathrm{geo}}[\mathrm{mm}]<0.12\end{cases}
$$

the typical values of which are presented in Table III for a single bunch, assuming the wake's first mode is dominant $\left(\kappa \simeq \kappa_{1}\right)$. The corresponding loaded gradient values are shown in Table III for three cases - each case corresponds to a different regime of the fluence. These results indicate that, due to the fluence dependence on pulse duration, high energy gain is available only for long structures, whereas the corresponding loaded gradient permissible is low.

Figure 5(a) shows the energy gain for the three fluence regimes in Eq. (5) as a function of group velocity. Although the energy gain is smaller as the structure is shorter, it is

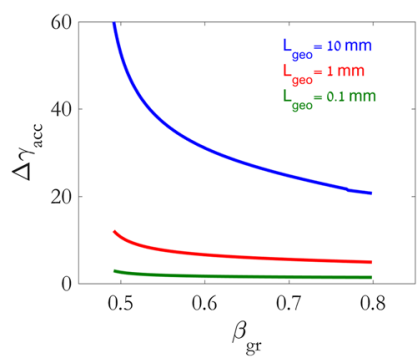

(a)

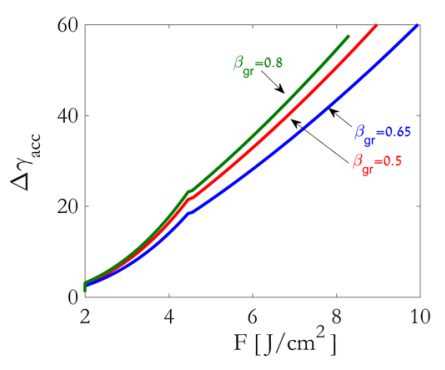

(b)
FIG. 5. Energy gain as a function of: (a) group velocity for the three fluence regimes, and (b) fluence for three values of group velocity. Although the energy gain is smaller for shorter structures, it is independent of the group velocity. Also, while the energy gain is a square root of the fluence (Eq. (A1), self consistent analysis indicates virtually linear dependence for $F>4.4 \mathrm{~J} / \mathrm{cm}^{2}$. independent of the group velocity. The latter's dependence on the structure's internal radius is shown in Appendix B. Figure 5(b) shows the energy gain for three values of group velocity, as a function of fluence. While the energy gain is a square root of the fluence [Eq. (A1)], the self consistent analysis indicates virtually linear dependence for $F>4.4 \mathrm{~J} / \mathrm{cm}^{2}$. Moreover, the energy gain discontinuity pertain to the discontinuity in the fluence (shown in Fig. 3).

\section{APPENDIX B: WAKE IN A PARTIALLY LOADED METALLIC CYLINDER}

In this Appendix we calculate the wake's weight functions $W_{s}$ of the envisaged configuration, shown in Fig. 1, by solving the nonhomogeneous dispersion relation in the presence of charge distribution in the structure.

The current density of a beam with a total charge $q$ and radius $R_{b}$, distributed at $r_{i}, z_{i}$, moving at a velocity $v$ is

$$
J_{z}(r, z, t)=-\frac{q v}{2 \pi r} \sum_{i} \delta\left(z-z_{i}-v t\right) \delta\left(r-r_{i}\right) .
$$

Let us assume a $\mathrm{TM}_{01}$ laser mode is copropagating with the beam in the z-direction with a phase velocity $\beta_{\mathrm{ph}}$ equal to the beam's velocity. The longitudinal electric field component of the mode is

$$
\begin{aligned}
E_{z}= & j \frac{\Gamma^{2}}{\varepsilon_{0} \omega} \exp \left(-j k_{z} z\right) \\
& \times \begin{cases}\frac{q}{4 \pi^{2}}\left[K_{0}(\Gamma r)+C_{1} I_{0}(\Gamma r)\right] & 0<r<R_{\mathrm{int}} \\
-\frac{\Lambda^{2}}{\Gamma^{2} \mu_{0} \varepsilon_{r}} C_{2} T_{0}(\Lambda r) & R_{\mathrm{int}}<r<R_{\mathrm{ext}}\end{cases}
\end{aligned}
$$

where $k_{z}=\omega /\left(c \beta_{\mathrm{ph}}\right), \quad \Gamma=\sqrt{k_{z}^{2}-(\omega / c)^{2}}$ and $\Lambda=$ $\sqrt{\varepsilon_{r}(\omega / c)^{2}-k_{z}^{2}}, K_{0}$ and $I_{0}$ are the modified Bessel function of the first and second kind respectively, $T_{0}(\Lambda r) \equiv J_{0}(\Lambda r) Y_{0}\left(\Lambda R_{\text {ext }}\right)-J_{0}\left(\Lambda R_{\text {ext }}\right) Y_{0}(\Lambda r)$, and $J_{n}$ and $Y_{n}$ are the $n$-th order Bessel function of the first and second kind respectively.

In the absence of charge, the homogeneous dispersion relation is

$$
\begin{aligned}
& D_{H}\left(\omega, k_{z}\right) \\
& \quad=\varepsilon_{r} T_{1}\left(\Lambda R_{\text {int }}\right) I_{0}\left(\Gamma R_{\text {int }}\right)+\frac{\Lambda}{\Gamma} T_{0}\left(\Lambda R_{\text {int }}\right) I_{1}\left(\Gamma R_{\text {int }}\right)
\end{aligned}
$$

where $T_{1}(\Lambda r) \equiv-J_{1}(\Lambda r) Y_{0}\left(\Lambda R_{\text {ext }}\right)+J_{0}\left(\Lambda R_{\text {ext }}\right) Y_{1}(\Lambda r)$. The coefficients $C_{1}, C_{2}$ in Eq. (B2) can be derived from the boundary conditions at the internal radius

$$
\begin{aligned}
C_{1} & =-\frac{\varepsilon_{r}}{\Gamma R_{\mathrm{int}}} \frac{T_{1}\left(\Lambda R_{\mathrm{int}}\right)}{I_{1}\left(\Gamma R_{\mathrm{int}}\right)} \frac{1}{D_{H}\left(\omega, k_{z}\right)} \\
C_{2} & =-\frac{\Gamma}{\Lambda} \frac{\mu_{0} q}{4 \pi^{2}} \frac{\varepsilon_{r}}{\Gamma R_{\mathrm{int}}} \frac{1}{D_{H}\left(\omega, k_{z}\right)} .
\end{aligned}
$$

In the time-domain, the averaged (over $r$ ) longitudinal electric field on the beam's radius $R_{b}$ is 


$$
\begin{aligned}
E_{z}(\tau=t-z / v) & =\int_{-\infty}^{\infty} d \omega \exp (j \omega t) \frac{2}{R_{b}^{2}} \int_{0}^{R_{b}} d r r E_{z}(\omega, r) \\
& =-j \frac{q}{4 \pi^{2} \varepsilon_{0} R_{\mathrm{int}}^{2}}\{\underbrace{\frac{4 R_{\mathrm{int}}^{2} \sqrt{\beta_{\mathrm{ph}}^{-2}-1}}{c R_{b}} \operatorname{Re} \int_{0}^{\infty} d \omega \exp (j \omega \tau) K_{1}\left(\Gamma R_{b}\right)}_{(1)}+\underbrace{\int_{-\infty}^{\infty} d \omega \exp (j \omega \tau) \frac{\mathrm{X}(\omega)}{\omega D_{H}\left(\omega, k_{z}\right)}}_{(2)}\}
\end{aligned}
$$

where $\mathrm{X}(\omega) \equiv 2 \varepsilon_{r} \mathrm{~T}_{1}\left(\Lambda \mathrm{R}_{\text {int }}\right) \mathrm{I}_{c}\left(\Gamma \mathrm{R}_{b}\right) / \mathrm{I}_{c}\left(\Gamma \mathrm{R}_{\text {int }}\right)$, and $I_{c}(x)=$ $2 I_{1}(x) / x$ for $x>0$ and 1 for $x=0$.

The first term represents the self-field of the particle, whereas the second term is the contribution of the structure. Using Cauchy's residue theorem for the second term, the wakefield is

$$
E_{\text {wake }}=E_{z}^{(2)}(\tau)=\kappa q \sum_{s} W_{s} \cos \left(\omega_{s} \tau\right) 2 h(\tau)
$$

where $h(t)$ is the Heaviside step function, $\kappa=1 /\left(2 \pi \varepsilon_{0} R_{\text {int }}^{2}\right)$ is the wake coefficient, and the wake's weight functions for the $s$ th mode are

$$
W_{s}=\left.\frac{\mathrm{X}(\omega)}{\omega \partial_{\omega} D_{H}\left(\omega, k_{z}\right)}\right|_{\omega=\omega_{s}}
$$

wherein the angular frequency $\omega_{s}$ is the solution of the homogeneous dispersion relation in Eq. (B3). Please note that $\sum_{s} W_{s}=1$, and that the first fundamental mode is $\omega_{\mathrm{L}}=\omega_{1}$.

As an example, Fig. 6(a) shows the wake's weight functions for 60 modes and their corresponding angular frequencies for $\lambda_{\mathrm{L}}=2 \mu \mathrm{m}, R_{\text {int }}=0.5 \lambda_{\mathrm{L}}, \varepsilon_{r}=2.1$ and the relativistic particle beam $(v=c)$. For this case, the phase velocity of the laser mode is $\beta_{\mathrm{ph}}=1$. As a result the weight functions are independent of the beam radius.

Please note that the projection of the wake on the fundamental (accelerating) mode $\left(\kappa_{1}=\kappa W_{1}\right)$ represents the maximum efficiency of the structure for a single bunch

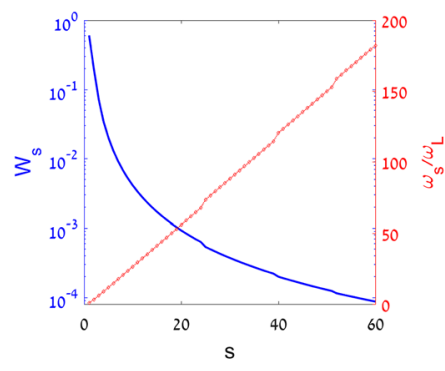

(a)

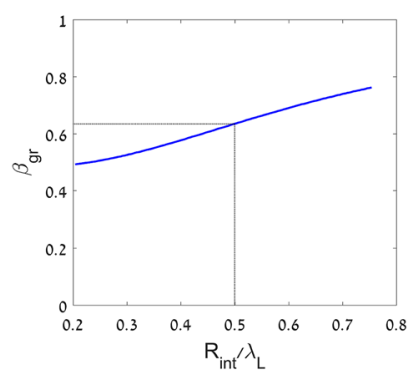

(b)
FIG. 6. For the parameters $\lambda_{\mathrm{L}}=2 \mu \mathrm{m}, \varepsilon_{r}=2.1$ and $\beta_{\mathrm{ph}}=1$ : (a) Wake's weight functions $W_{s}$ (blue) for the first 60 modes and their corresponding angular frequencies $\omega_{s}$ (red) for $R_{\text {int }}=0.5 \lambda_{\mathrm{L}}$. (b) Group velocity of the envisaged structure as a function of the internal radius.

$$
\eta_{1, \max }=\frac{\kappa_{1}}{\kappa}=W_{1}
$$

For the self-consistent formulation, the group velocity dependence on the radii must be presented. Despite the fact that in this section we develop the nonhomogeneous solution of the wake, it warrants to point out that this solution contains information about the homogeneous solution, specifically about the group velocity in the system,

$$
\beta_{\mathrm{gr}}\left(R_{\mathrm{int}}\right)=-\left.\frac{1}{c} \frac{\partial D_{H}\left(\omega, k_{z}\right) / \partial k_{z}}{\partial D_{H}\left(\omega, k_{z}\right) / \partial \omega}\right|_{\frac{\omega=\omega_{\mathrm{L}}}{k_{z}=\frac{\omega_{\mathrm{L}}}{c}}} .
$$

Figure 6(b) shows the dependence of the group velocity on the internal radius. For each internal radius, the external radius is calculated via the dispersion relation, and then the group velocity is derived.

\section{APPENDIX C: LOADED GRADIENT IN THE REDUCED AND UNREDUCED CASES}

The set of Eqs. (4)-(7), describing the bunch's charge $q$ in the reduced case, could be written in terms of the charge in the un-reduced case [Eq. (9)], namely

$$
q^{(\mathrm{R})}=\frac{1}{1-W_{1}} q^{(\mathrm{UR})} .
$$

Keeping in mind that the effective field in the dielectric [Eq. (3)]

$$
E_{\mathrm{eff}}=\frac{\sqrt{2 \eta_{0} \varepsilon_{r}}}{\frac{1}{2} \frac{\omega_{\mathrm{L}}}{c} R_{\mathrm{int}}} \sqrt{\frac{F\left(\tau_{\mathrm{p}}\right)}{\tau_{\mathrm{p}}}}
$$

is equal to $G_{0}^{(\mathrm{UR})}$ in the unreduced case, and to

$$
E_{\text {eff }}=G_{0}^{(\mathrm{R})}-\kappa_{1} q^{(\mathrm{R})}
$$

in the reduced case, in what follows we show that the loaded gradient in both cases is the same.

Substituting Eqs. (C1) and (C2), the loaded gradient in the unreduced case is 


$$
\begin{aligned}
G_{\text {Loaded }}^{(\mathrm{UR})} & =G_{0}^{(\mathrm{UR})}-\kappa q^{(\mathrm{UR})} \\
& =E_{\text {eff }}-\kappa q^{(\mathrm{R})}\left(1-W_{1}\right)=E_{\text {eff }}-\kappa q^{(\mathrm{R})}+\kappa_{1} q^{(\mathrm{R})} .
\end{aligned}
$$

Substituting Eq. (C3) to the former results in

$$
G_{\text {Loaded }}^{(\mathrm{UR})}=G_{0}^{(\mathrm{R})}-\kappa q^{(\mathrm{R})}=G_{\text {Loaded }}^{(\mathrm{R})},
$$

implying that the loaded gradients are the same for the reduced and unreduced cases. Therefore, the unloaded gradient in the reduced case is expected to be higher, and so does the amount of charge in the single bunch-as was shown in Table II.

[1] J. Wang and G. Loew, Field emission and RF breakdown in high-gradient room-temperature linac structures, Report No. Slac-Pub 7684, 1997, p. 28.

[2] A. Hanuka, L. Schächter, K. P. Wootton, Z. Wu, K. Soong, I. V. Makasyuk, and R. J. England, Cumulative damage of ultrafast laser pulses, in Proceedings of IPAC16 (2016), p. 1, DOI: 10.18429/JACoW-IPAC2016THPOW054.

[3] K. Soong, R. Byer, C. McGuinness, E. Peralta, and E. Colby, Experimental determination of damage threshold characteristics of IR compatible optical materials, in Proceedings of the 24th Particle Accelerator Conference, PAC-2011, New York, 2011 (IEEE, New York, 2011), p. $277-279$.

[4] J. T. Seeman, The Stanford linear collider, AIP Conf. Proc. 2, 249 (1992).

[5] R. J. England et al., Dielectric laser accelerators, Rev. Mod. Phys. 86, 1337 (2014).

[6] L. Schächter, Energy recovery in an optical linear collider, Phys. Rev. E 70, 016504 (2004).

[7] V. Karagodsky, D. Schieber, and L. Schächter, Enhancing $\mathrm{X}$-Ray Generation by Electron-Beam-Laser Interaction in an Optical Bragg Structure, Phys. Rev. Lett. 104, 024801 (2010).

[8] R. J. England, R. J. Noble, B. Fahimian, B. Loo, E. Abel, A. Hanuka, and L. Schachter, Conceptual layout for a wafer-scale dielectric laser accelerator, AIP Conf. Proc. 1777, 060002 (2016).

[9] X. Lin, Photonic band gap fiber accelerator, Phys. Rev. ST Accel. Beams 4, 051301 (2001).

[10] A. Mizrahi and L. Schächter, Optical Bragg accelerators, Phys. Rev. E 70, 016505 (2004).

[11] B. M. Cowan, Photonic crystal laser-driven accelerator structures, Ph.D. thesis, Stanford, 2007.
[12] Z. Wu, R. J. England, C. K. Ng, B. Cowan, C. McGuinness, C. Lee, M. Qi, and S. Tantawi, Coupling power into accelerating mode of a three-dimensional silicon woodpile photonic band-gap waveguide, Phys. Rev. ST Accel. Beams 17, 081301 (2014).

[13] J. Harrison, Y. Hwang, O. Paydar, J. Wu, E. Threlkeld, J. Rosenzweig, P. Musumeci, and R. Candler, High-gradient microelectromechanical system quadrupole electromagnets for particle beam focusing and steering, Phys. Rev. ST Accel. Beams 18, 023501 (2015).

[14] E. A. Peralta, K. Soong, R. J. England, E. R. Colby, Z. Wu, B. Montazeri, C. McGuinness, J. McNeur, K. J. Leedle, D. Walz, E. B. Sozer, B. Cowan, B. Schwartz, G. Travish, and R. L. Byer, Demonstration of electron acceleration in a laser-driven dielectric microstructure, Nature (London) 503, 91 (2013).

[15] K. Wootton, Z. Wu, B. Cowan, A. Hanuka, I. Makasyuk, E. Peralta, K. Soong, R. Byer, and R. England, Demonstration of acceleration of relativistic electrons at a dielectric microstructure using femtosecond laser pulses, Opt. Lett. 41, 2696 (2016).

[16] B. C. Stuart, M. D. Feit, A. M. Rubenchik, B. W. Shore, and M. D. Perry, Laser-Induced Damage in Dielectrics with Nanosecond to Subpicosecond Pulses, Phys. Rev. Lett. 74, 2248 (1995).

[17] A. Grudiev, S. Calatroni, and W. Wuensch, New local field quantity describing the high gradient limit of accelerating structures, Phys. Rev. ST Accel. Beams 12, 102001 (2009).

[18] L. Schächter, R. L. Byer, and R. H. Siemann, Wake field in dielectric acceleration structures, Phys. Rev. E 68, 036502 (2003).

[19] R. H. Siemann, Energy efficiency of laser driven, structure based accelerators, Phys. Rev. ST Accel. Beams 7, 061303 (2004).

[20] A. Hanuka and L. Schächter, Bragg accelerator optimization, High Power Laser Sci. Eng. 2, e24 (2014).

[21] M. Rosing and W. Gai, Longitudinal- and transverse-wakefield effects in dielectric structures, Phys. Rev. D 42, 1829 (1990).

[22] L. Schachter, Beam-Wave Interaction in Periodic and Quasi-Periodic Structures (Springer, Berlin, Heidelberg, 2011), DOI: 10.1007/978-3-642-19848-9.

[23] D. Cesar, S. Custodio, J. Maxson, P. Musumeci, X. Shen, E. Threlkeld, R. J. England, A. Hanuka, I. V. Makasyuk, E. A. Peralta, K. P. Wootton, and Z. Wu, Nonlinear response in high-field dielectric laser accelerators, arXiv:1707.02364.

[24] L. Schächter, R. L. Byer, and R. H. Siemann, Optical accelerator: scaling laws and figures of merit, AIP Conf. Proc. 647, 310 (2002).

[25] A. Hanuka and L. Schachter, Operation regimes of a dielectric laser accelerator, Nucl. Instrum. Methods Phys. Res., Sect. A 888, 147 (2018). 